\title{
DINÂMICA DA DISTRIBUIÇÃO E DEGRADAÇÃO DE ATRAZINA EM ARGISSOLO VERMELHO-AMARELO SOB CONDIÇÕES DE CLIMA TROPICAL ÚMIDO ${ }^{(1)}$
}

\author{
Fábio Veríssimo Correia ${ }^{(2)} \&$ Tomaz Langenbach $^{(3)}$
}

\begin{abstract}
RESUMO
A biodegradabilidade e a distribuição dos agrotóxicos no ambiente determinam o seu potencial poluidor. O risco de contaminação do herbicida atrazina, em Argissolo Vermelho-Amarelo, foi estudado em experimentos de campo complementados com a utilização do sistema de microcosmo. $\mathrm{O}$ microcosmo permitiu utilizar ${ }^{14} \mathrm{C}$-atrazina em condições controladas e determinar a volatilização $(0,33 \%)$, mineralização $(0,25 \%)$ e lixiviação (4 a $11 \%)$, parâmetros difíceis de determinar no campo. Verificou-se que $90 \%$ da radioatividade ficou no solo, encontrando-se cerca de $75 \%$ nos primeiros $5 \mathrm{~cm}$. O experimento de campo foi feito na época seca, permitindo a simulação de chuvas de verão. Os resultados mostraram que, 90 dias após a aplicação, a atrazina foi encontrada a $50 \mathrm{~cm}$ de profundidade, podendo ter atingido as águas do subsolo. A atrazina deslocada pelo escoamento do excesso de água encontrava-se principalmente na fase líquida, $1,6 \%$ do valor aplicado, enquanto $0,4 \%$ foi adsorvido nas partículas erodidas. Os valores de atrazina na água do escorrimento foram maiores dois dias após aplicação, decrescendo a menos de um décimo após 15 dias. Os resultados evidenciam que a aplicação do herbicida deve ser evitada quando houver previsão de chuvas, considerando o potencial poluidor de águas superficiais e subterrâneas neste tipo de solo.
\end{abstract}

Termos de indexação: escorrimento superficial, erosão, poluição do solo, lixiviação, volatilização, adsorção, mineralização, herbicida.

${ }^{(1)}$ Recebido para publicação em janeiro de 2004 e aprovado em fevereiro de 2005.

(2) Pesquisador do Instituto de Microbiologia, Universidade Federal do Rio de Janeiro - UFRJ. Cidade Universitária Ilha do Fundão, Bloco I, Sala 29, CEP 21941-970 Rio de Janeiro (RJ). E-mail: fabioverissimo@yahoo.com.br

(3) Professor Adjunto do Instituto de Microbiologia, UFRJ. E-mail: langenbach@micro.ufrj.br 


\title{
SUMMARY: DISTRIBUTION AND DECOMPOSITION DYNAMICS OF ATRAZINE IN AN ULTISOL UNDER WET TROPICAL CLIMATE CONDITIONS
}

\begin{abstract}
The biodegradability and distribution of pesticides in the environment determine their pollution potential. The risk of contamination with atrazine herbicide was studied in an Ultisol under field conditions and complemented with a microcosm system study. This system allowed the use of ${ }^{14} \mathrm{C}$-atrazine under controlled conditions and to determine of the volatilization $(0.33 \%)$, mineralization $(0.25 \%)$ and leaching (4 to $11 \%)$ parameters, which are difficult to evaluate in the field. Ninety percent of the radioactivity remained in the soil, with $75 \%$ in the first $5 \mathrm{~cm}$. The field experiment was carried out during the dry season, allowing the simulation of summer rains. Results showed that 90 days after the application, atrazine was found at a depth of $50 \mathrm{~cm}$ and could well have reached the groundwater. Atrazine lost by runoff was found mainly in the liquid phase (1.6\% of total applied), whereas $0.4 \%$ was adsorbed to the eroded particles. The atrazine concentration in the runoff was higher on the second day after application, decreasing to less than a tenth of that value after 15 days. These results suggest that application of this herbicide needs to be avoided when rain is forecast due to the pollution potential for ground and surface water in this kind of soil.
\end{abstract}

Index terms: runoff, erosion, soil pollution, herbicide, leaching, volatilization, adsorption, mineralization, herbicide.

\section{INTRODUÇÃO}

Nas últimas décadas, intensificou-se o uso do monocultivo de milho e soja em grandes extensões territoriais brasileiras (Goedert, 1983). Estas culturas demandam elevadas quantidades de agroquímicos, comprometendo a qualidade ambiental pela contaminação do solo e água (Connell, 1990; Dörfler et al., 1996). Atualmente, o Brasil encontra-se entre os dez maiores consumidores de agrotóxicos no mundo, com cerca de 250 ingredientes ativos registrados no Instituto Brasileiro de Meio Ambiente. Apesar da legislação restritiva ao uso de produtos organoclorados, o consumo de agrotóxicos triplicou nos últimos 15 anos e continua crescendo, motivo de grande apreensão (SINDAG, 1998).

Os pesticidas atingem o solo através da aplicação direta sobre sua superfície; indiretamente, quando aplicados sobre as plantas, ou por contaminação aérea. No solo, os processos de adsorção, lixiviação, volatilização e degradação biótica e abiótica, assim como a translocação na planta, determinam o grau de biodisponibilidade, a forma e a intensidade dos efeitos nocivos da poluição (Schroll et al., 1992).

$\mathrm{Na}$ região sudeste do Brasil freqüentemente ocorrem chuvas torrenciais no verão, fazendo com que uma parte da água se infiltre no solo e a porção excedente à capacidade de infiltração escoe superficialmente, promovendo erosão (Bertoni \& Lombardi-Neto, 1993). O transporte de pesticidas na água que se move sobre a superfície do solo, chamado de escoamento superficial, tem sido considerado como um dos principais meios de contaminação de rios e lagos (Gaynor et al., 1992; Lerch \& Blanchard, 2003). Estudos têm mostrado que essas perdas de herbicidas geralmente variam de 1 a $5 \%$, dependendo das práticas culturais, solo, dimensão da área, declividade, extensão do declive, cobertura, umidade do solo e das propriedades dos herbicidas (Belamie \& Gouy, 1992; Carluer et al., 1996; Patty et al., 1997). O movimento da água carreia substâncias solúveis ou adsorvidas às partículas de solos erodidos.

Uma vez que o uso de pesticidas se distribui em grandes áreas agrícolas, devem-se priorizar as práticas preventivas da poluição das águas em detrimento de métodos de remoção desses contaminantes, como a biorremediação, por serem de alto custo e exeqüibilidade limitada.

Inúmeros estudos têm identificado problema de poluição de águas superficiais e subterrâneas decorrentes do uso de pesticidas em regiões temperadas (Solomon et al.,1995; Kreuger, 1998), porém o destino destes produtos em solos sob condições tropicais é pouco conhecido (Langenbach et al., 2000; Laabs et al., 2000; Langenbach et al., 2001; Reichenberg et al., 2002).

Como os herbicidas são os agrotóxicos mais utilizados (55\% dos pesticidas) e de difícil substituição, há necessidade de intensificar estudos com eles. O herbicida atrazina (2-cloro-4-etilamino6-isopropilamino-s-triazina) faz parte da família das triazinas, intensamente utilizadas no Brasil e no mundo, sendo freqüentemente detectado como o principal poluente orgânico de águas subterrâneas e superficiais em vários países (Dörfler et al., 1997).

O trabalho teve dois objetivos: o primeiro foi estudar, em condições de laboratório, a distribuição do herbicida atrazina por volatilização, lixiviação, adsorção ao longo do perfil do solo e mineralização, possibilitando um balanço de massa. Para rastrear esta molécula e seus produtos de degradação, foi 
necessário utilizar um sistema fechado de microcosmo com ${ }^{14} \mathrm{C}$-atrazina. Esta abordagem permite a simulação de condições climáticas com obtenção de resultados representativos do ambiente (Österreicher-Cunha et al., 2003); o segundo objetivo foi estudar o escorrimento superficial em experimento de campo, pela dificuldade de simular, de forma representativa, o ambiente com microcosmo. No experimento, procurou-se, maximizar o processo de escorrimento superficial com aplicação de regime curto de enxurradas muito fortes, numa cultura de pouca cobertura do solo, como o milho (Hernani et al., 1997). Acredita-se que as informações obtidas poderão contribuir como subsídios importantes para avaliação do risco de contaminação de águas superficiais e subterrâneas com atrazina.

\section{MATERIAL E MÉTODOS}

\section{Descrição da área}

O experimento foi efetuado em Argissolo Vermelho-Amarelo distrófico típico (adaptado de Ramos et al., 1973), localizado na área experimental do Departamento de Fitotecnia da Universidade Federal Rural do Rio de Janeiro, no município de Seropédica (RJ), numa área de $800 \mathrm{~m}^{2}$, com $12 \%$ de declividade. A análise granulométrica do solo foi feita pelo método de pipeta (Day, 1965) e o fracionamento da areia de acordo com o sistema de classificação da USDA (USDA, 1975), nas camadas de 0 a 10 e de 10 a $50 \mathrm{~cm}$ de profundidade (Quadro 1). Com base na análise do solo, efetuaram-se as aplicações das doses de calcário, para correção da acidez até $\mathrm{pH}$ 6,5, e fertilização para o cultivo da cultura do milho (Embrapa, 1997).

\section{Experimento de laboratório}

Montagem do microcosmo - O solo foi coletado 30 dias após seu preparo em amostras indeformadas, com o auxílio de cilindros de aço inox de $30 \mathrm{~cm}$ de altura e $15 \mathrm{~cm}$ de diâmetro, em quatro repetições, escolhidas aleatoriamente em diferentes pontos da área. As amostras foram transportadas para o laboratório para montagem do sistema de microcosmo (Figura 1), o qual funciona com um fluxo de ar controlado por um rotâmetro regulado a $17 \mathrm{~L} \mathrm{~h}^{-1}$, mantido por um sistema de bombas. A simulação de vento é promovida por uma hélice acoplada à tampa, alcançando aproximadamente $1 \mathrm{~m} \mathrm{~s}^{-1}$. O fluxo de ar perpassa duas armadilhas posicionadas em série, que contêm espuma de poliuretano em frascos de $500 \mathrm{~mL}$ para captação dos compostos voláteis. Em seguida, o fluxo atravessa duas armadilhas químicas com $20 \mathrm{~mL}$ da mistura de etanolamina e etilenoglicolmonobutileter (1:1) para captar o ${ }^{14} \mathrm{CO}_{2}$. As amostras nas armadilhas foram colhidas e trocadas duas vezes por semana. O solo foi saturado para padronizar o teor de umidade e, após uma semana de repouso, efetuou-se a aplicação da ${ }^{14} \mathrm{C}$-atrazina. $\mathrm{O}$ experimento foi mantido com índice pluviométrico de verão (272 mm mês $\left.{ }^{-1}\right)$, a partir da segunda semana após aplicação do produto. A simulação de chuva foi feita por aplicação de água três vezes por semana distribuída homogeneamente sobre a superfície do solo. O sistema de microcosmo foi mantido em temperatura ambiente, que variou de 23 a $40^{\circ} \mathrm{C}$. Um sistema de bombas de sucção promovia um vácuo de $75 \mathrm{~mm} \mathrm{Hg}$, simulando as forças de sucção da água pelo solo. O lixiviado foi coletado uma vez por semana em frascos e acondicionado a $4{ }^{\circ} \mathrm{C}$ para posterior determinação da radioatividade. $\mathrm{O}$ experimento teve a duração de 60 dias e foi realizado com quatro repetições.

Preparo da solução de ${ }^{14} \mathrm{C}$-atrazina - A molécula marcada com ${ }^{14} \mathrm{C}$ no anel da atrazina foi purificada a $99 \%$ pela técnica de cromatografia de camada fina (TLC) com placa Merk (AL 20 x $20 \mathrm{~cm}$ Silicagel $60 \mathrm{~F}_{254}$ ), utilizando como solvente a solução diclorometano:metanol (95:5/v:v). A faixa da placa ocupada pela atrazina purificada foi recortada e eluída com acetona. O material coletado foi transferido para um vial que continha $19,92 \mathrm{mg}$ de atrazina (Gesaprim 500) e ajustado a $2 \mathrm{~mL}$ com água. Desta solução, com o auxílio de uma seringa Hamilton, foram aplicados, homogeneamente, na superfície do solo de cada microcosmo, os volumes de $500,450,450$ e $400 \mu \mathrm{L}$, que continham, respectivamente, $182.000,164.000,164.000 \mathrm{e}$

Quadro 1. Análise granulométrica do Argissolo Vermelho-Amarelo distrófico típico, Seropédica (RJ)

\begin{tabular}{|c|c|c|c|c|c|c|c|c|c|c|}
\hline \multirow{2}{*}{ Profundidade } & \multirow{2}{*}{ Argila } & \multirow{2}{*}{ Silte } & \multicolumn{6}{|c|}{ Areia } & \multirow{2}{*}{$\mathbf{p H}$} & \multirow{2}{*}{ MO } \\
\hline & & & Total & M. grossa & Grossa & Média & Fina & M. fina & & \\
\hline $\mathrm{cm}$ & & & & 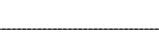 & $\%$ & & & $\underline{-}$ & & $\mathrm{g} \mathrm{kg}^{-1}$ \\
\hline $0-10$ & 24,00 & 10,49 & 65,47 & 6,98 & 16,35 & 18,40 & 19,08 & 4,66 & 5,77 & 28,0 \\
\hline $10-50$ & 28,76 & 10,60 & 60,45 & 4,81 & 13,43 & 17,58 & 19,59 & 5,08 & 5,67 & 8,33 \\
\hline
\end{tabular}

Valores representam médias de três repetições. 


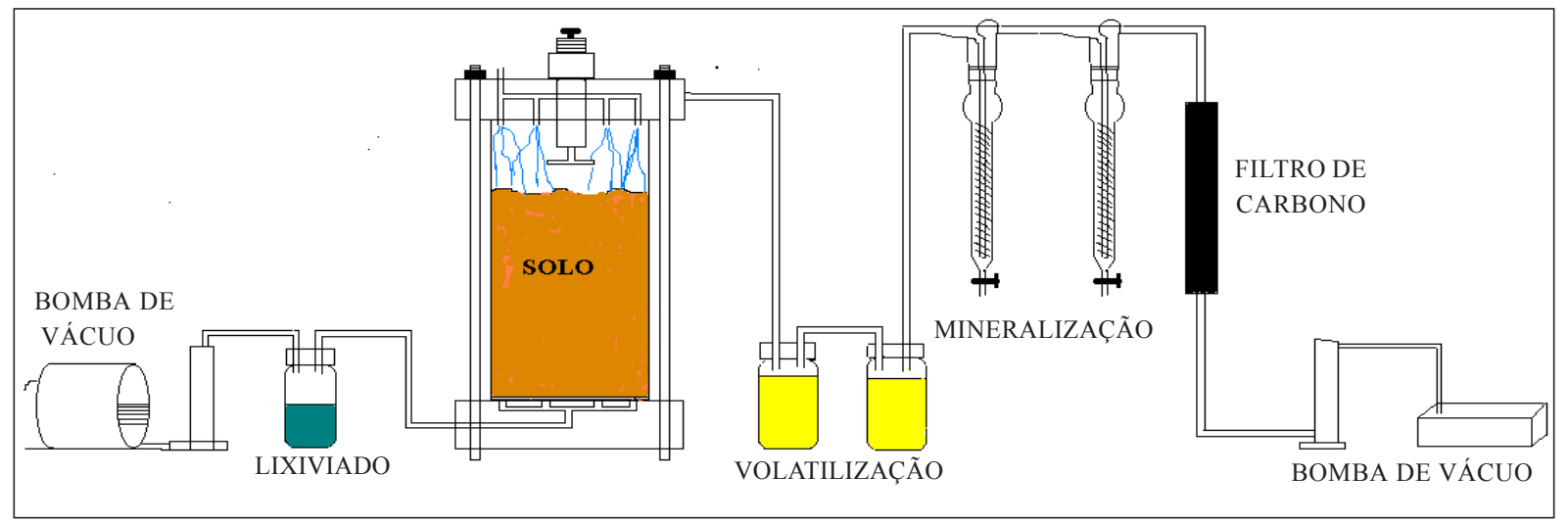

Figura 1. Esquema do microcosmo.

145.000 Bq. Os cálculos foram feitos de modo que cada microcosmo recebesse aproximadamente a concentração agronômica recomendada de $3 \mathrm{~kg} \mathrm{ha}{ }^{-1}$ i.a.

Determinação da radioatividade - Os produtos radioativos voláteis retidos na espuma de poliuretano foram extraídos com $200 \mathrm{~mL}$ de acetona, concentrados à secura e ressuspendidos em volumes ajustados de $5 \mathrm{~mL}$ de acetona. Esta alíquota foi adicionada a $10 \mathrm{~mL}$ de solução cintiladora ( $4 \mathrm{~g}$ difenil-ozalato, $0,25 \mathrm{~g}$ [2,26-p-Penil-bis-(5feniloxazol)], $333 \mathrm{~mL}$ Triton X 100 e completada com tolueno até $1 \mathrm{~L}$ ) e medida no cintilador líquido da Beckman modelo 4100. O ${ }^{14} \mathrm{CO}_{2}$ e a radioatividade do lixiviado foram coletados em alíquotas de $5 \mathrm{~mL}$, adicionados a $10 \mathrm{~mL}$ de solução cintiladora e medidos no cintilador Beckman 4100. A radioatividade no solo foi medida ao final dos dois meses de experimento. O solo foi retirado do microcosmo e, em seguida, foi fatiado em camadas de $1 \mathrm{~cm}$ nos primeiros $5 \mathrm{~cm}$ superficiais. As camadas subjacentes até os $30 \mathrm{~cm}$ de profundidade foram fatiadas de 5 em $5 \mathrm{~cm}$. Cada camada foi seca ao ar, pesada e cuidadosamente homogeneizada. Três réplicas das amostras de solo entre 0,5 e $1 \mathrm{~g}$ foram pesadas em balança analítica e, posteriormente, queimadas em Oxidizer Zinsser (Oximat 500). $\mathrm{O}^{{ }^{14}} \mathrm{CO}_{2}$ formado foi absorvido por uma solução de cintilação aquasolv (1 L solução cintiladora + $666 \mathrm{~mL}$ metanol + $416 \mathrm{~mL}$ etanolamina) e contado no cintilador. Os valores de dpm foram relacionados com a radioatividade total aplicada em cada microcosmo e expressos em percentagem. Os valores apresentados foram as médias dos quatro microcosmos e o desvio-padrão está representado pelas barras nas figuras.

\section{Experimento de campo}

Preparo da área - A área foi dividida em 18 parcelas experimentais de $6 \mathrm{~m}$ de comprimento e $4 \mathrm{~m}$ de largura. $\mathrm{O}$ delineamento experimental referente aos diferentes tempos de amostragem (2, 15, 30, 60 e 90 dias após a aplicação do herbicida) foi feito em triplicata e inteiramente casualizado. Dentro de cada parcela foi delimitada uma área de $2,25 \mathrm{~m}^{2}(1,5 \times 1,5)$ para aplicação do herbicida atrazina (Gesaprim 500), 30 dias após a semeadura, na dose recomendada de $6 \mathrm{~L} \mathrm{ha}^{-1}$.

Regime de irrigação - $\mathrm{O}$ experimento na época seca (entre maio e setembro) possibilitou a irrigação da área de forma controlada, simulando o regime de chuva de verão típico da região $\left(200 \mathrm{~mm}\right.$ mês $\left.{ }^{-1}\right)$. A irrigação foi realizada manualmente, com um bico pulverizador acoplado à ponta de uma mangueira e foi iniciada a partir do segundo dia da aplicação do herbicida. A quantidade de água foi calculada de acordo com área e tempo de aplicação, totalizando aproximadamente $50 \mathrm{~mm}$ de água por semana, distribuída em três aplicações semanais.

Coleta de amostras de água do escorrimento superficial - Aos 2, 15, 30, 60 e 90 dias da aplicação do herbicida, colocou-se uma calha de metal de $1 \mathrm{~m}$ de comprimento por $0,75 \mathrm{~m}$ de largura, cravada no solo, para coleta da água escorrida superficialmente nas parcelas. A simulação de chuvas torrenciais foi de $780 \mathrm{~mm} \mathrm{~h}^{-1}$, com fluxo constante por $4 \mathrm{~min}$, garantindo a saturação do solo e o escorrimento superficial. O excedente de água da calha de metal foi coletado em recipientes posicionados logo abaixo da área da calha, homogeneizado e 1,0 L deste material foi guardado a $4{ }^{\circ} \mathrm{C}$, e procedeu-se em menos de $48 \mathrm{~h}$ a análise de atrazina.

Coleta de amostras de solo - Após a simulação de chuvas torrenciais e coleta de amostras da água do escorrimento superficial de cada parcela, foram coletadas amostras de solo de 5 em $5 \mathrm{~cm}$ até à profundidade de $50 \mathrm{~cm}$ com o auxílio de um cilindro de volume conhecido $\left(355 \mathrm{~cm}^{3}\right)$. De cada camada foram preparadas amostras compostas de $200 \mathrm{~g}$. A amostragem em cada parcela foi feita em triplicata.

Análise de atrazina - Solo: Foram pesados $50 \mathrm{~g}$ de solo e adicionados $100 \mathrm{~mL}$ de acetona para extração da atrazina. A suspensão foi agitada durante 20 min e, a seguir, tratada com banho de 
ultra-som, durante $10 \mathrm{~min}$, para solubilização dos resíduos e metabolitos. Em seguida, foi filtrada em papel de filtro Whatman $\mathrm{n}^{0} 4$ e concentrada até à secura. A amostra foi ressuspendida em $100 \mathrm{~mL}$ de solução metanol:água (85:15 v/v) e transferida para um funil de decantação, no qual se efetuou a purificação com $30 \mathrm{~mL}$ de diclorometano. Após a agitação e decantação, a fração inferior do funil (diclorometano) foi separada. O sobrenadante contido no funil foi tratado mais duas vezes com diclorometano, conforme já descrito. Todas as frações de diclorometano foram reunidas e concentradas no evaporador rotativo. Em seguida, foram ressuspendidas em $10 \mathrm{~mL}$ de metanol. Desta porção foi retirada uma alíquota de $2 \mathrm{~mL}$, que foi concentrada até, aproximadamente, $0,5 \mathrm{~mL}$ e aplicada, em seguida, numa coluna de fase reversa de $\mathrm{C}_{18}$ (extrator de fase sólida) previamente ativada. A amostra da coluna foi eluída com 5,0 mL de água e o volume percolado foi descartado. Posteriormente, foram adicionados 5,0 mL de solução diclorometano: metanol (7:3 v/v) cujo eluato que continha os resíduos foi recolhido em balão volumétrico, concentrado à secura e ressuspendido com $5,0 \mathrm{~mL}$ de solução acetonitrila : água (8:2 v/v). O método de extração e purificação foi adaptado dos métodos utilizados por Huang \& Pignatello (1990) e Balinova \& Balinov (1991), quando foram testados diferentes solventes e diferentes tempos de agitação. A eficiência na recuperação do herbicida pelo método variou de 75 a $85 \%$.

Água do escorrimento: Duzentos mililitros da água de escorrimento foram filtradas em papel Whatman $\mathrm{n}^{\circ} 4$, tendo sido os sedimentos retidos guardados no filtro. A solução filtrada foi submetida ao mesmo processo de purificação com diclorometano descrito para as amostras de solo.

Sedimentos: Dos sedimentos retidos da filtração dos $200 \mathrm{~mL}$ da água de escorrimento foi extraída a suspensão atrazina com acetona. A porção líquida foi submetida ao mesmo processo de extração e purificação aplicado às amostras de solo.

Análise em cromatografia líquida de alta eficiência (CLAE) - Os extratos de solo, água e sedimentos purificados foram filtrados em membrana $(0,22 \mu \mathrm{m}$ - Millipore GV Millex / polietileno) e transferidos para frascos de CLAE (Huang \& Pignatello, 1990; Balinova \& Balinov, 1991). A concentração de atrazina foi medida por CLAE, usando detector UV, a $254 \mathrm{~nm}$, após separação em coluna de fase reversa $\mathrm{RP}_{8}(5 \mu \mathrm{m}$, $250 \mathrm{~mm} \times 4 \mathrm{~mm}$ ), utilizando-se como fase móvel metanol:água deionizada (60:40 v/v). Com o fluxo de $0,5 \mathrm{~mL} \mathrm{~min}^{-1}$ a coluna foi mantida em temperatura ambiente $\left(26^{\circ} \mathrm{C}\right)$. Todas as amostras foram analisadas nessas condições, com o volume de injeção de $20 \mu \mathrm{L}$. A determinação da concentração destas substâncias foi realizada por meio de uma curva de calibração construída com atrazina padrão (grau de pureza 99 \%). A detecção de atrazina foi confirmada em mais duas corridas em fase móvel de acetonitrila:água com diferentes gradientes $(50: 50,65: 35 \mathrm{v} / \mathrm{v})$, sob as mesmas condições anteriores.

\section{RESULTADOS E DISCUSSÃO}

\section{Experimento no microcosmo}

Volatilização de atrazina - Os maiores valores de produtos radioativos voláteis foram medidos nos primeiros dias de experimento, atingindo, no oitavo dia, picos de aproximadamente $0,02 \%$ do total aplicado e decrescendo gradativamente com o decorrer dos 60 dias (Figura 2). O pico apresentado no oitavo dia pode ser atribuído ao início da aplicação de água na simulação de chuvas no microcosmo. Os resultados coincidem com as observações de Glotfelty et al. (1984) e Langenbach et al. (2000), segundo os quais o processo de volatilização foi intensificado no período subseqüente à aplicação de água ao solo, que talvez tenha promovido a perda do composto pelo arraste de vapor, fenômeno chamado de codestilação.

Em condições de campo, a maior incidência de radiação solar e o conseqüente aumento na temperatura do solo podem fornecer valores maiores de volatilização do que os medidos no sistema de microcosmo (Glotfelty et al., 1989).

Essa abordagem permite compreender e comparar os parâmetros determinantes no processo de volatilização. O decréscimo nos valores de volatilização ocorre com a redução do teor de atrazina disponível na superfície à medida que progressivamente são estabelecidas ligações de adsorção menos reversíveis, perdas por degradação e deslocamento de pesticidas para camadas mais profundas por lixiviação (Clay \& Koskinen, 1990; Roy \& Krapac, 1994).

Segundo o balanço de massa apresentado na figura 3 , aproximadamente $0,33 \%$ do total de ${ }^{14} \mathrm{C}$ atrazina aplicada na superfície do solo foi volatilizado. O reduzido percentual de volatilização também foi influenciado pelo baixo valor da pressão de vapor da atrazina $\left(3,0 \times 10^{-7} \mathrm{~mm} \mathrm{Hg}\right)$. Embora os percentuais sejam reduzidos, as grandes quantidades de pesticidas aplicados em extensas áreas agrícolas permitem que as moléculas volatilizadas sejam deslocadas pelos ventos para ambientes onde nunca foram utilizados, poluindo ecossistemas naturais e áreas urbanas (Polkowska et al., 2002).

Degradação de atrazina - Os dados do balanço de massa apresentados na figura 3 indicam que a dissipação da atrazina por mineralização foi muito baixa neste solo, comportamento que, segundo Adams \& Thurman (1991), ainda é mais reduzido à 


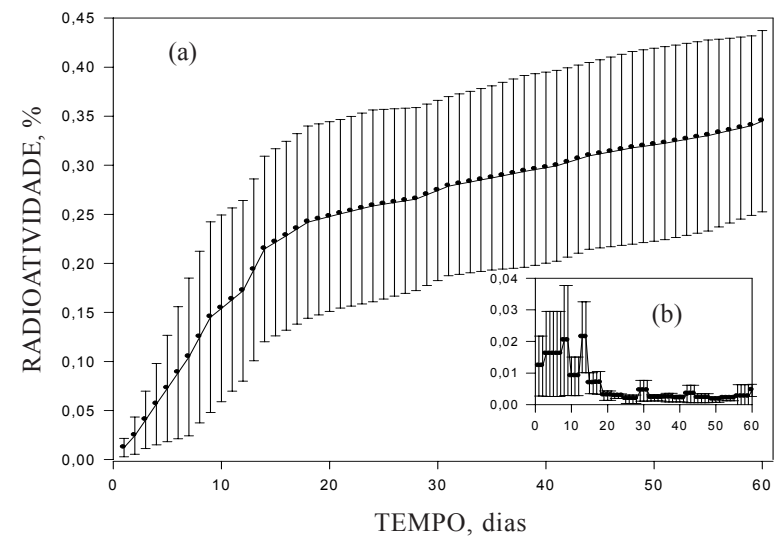

Figura 2. Percentagem de produtos radioativos voláteis liberados nos 60 dias de experimento (média dos 4 microcosmos) ( $\mathrm{a}=\%$ acumulada, $\mathbf{b}=\%$ diária). As barras verticais indicam o desvio-padrão.

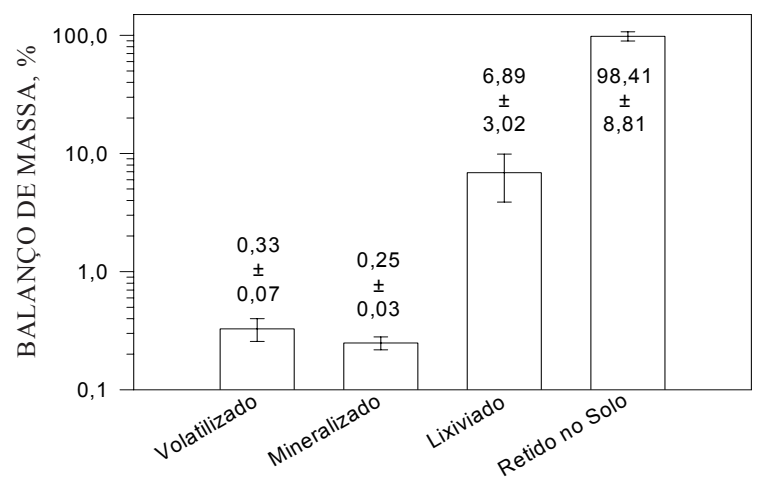

Figura 3. Balanço de massa (média de quatro repetições e desvio-padrão) de ${ }^{14} \mathrm{C}$-atrazina aplicada a Argissolo Vermelho-Amarelo após 60 dias de experimento.

medida que o herbicida lixiviado atinge o subsolo. Segundo o critério do Instituto Brasileiro de Meio Ambiente (1990), o valor de 0,25\% de ${ }^{14} \mathrm{CO}_{2}$ mineralizado durante 60 dias classifica a atrazina como um composto de persistência alta no presente estudo. Seiler et al. (1992) e Han \& New (1994) apresentaram valores de degradação da atrazina entre 0,005 e $30 \%$ em períodos menores que 90 dias. Peixoto (1998) observou, num Latossolo VermelhoEscuro, o valor total de $0,12 \%$ de ${ }^{14} \mathrm{CO}_{2}$, enquanto Nakagawa et al. (1995), em Latossolo VermelhoEscuro e Glei Húmico, verificaram valores bem maiores de ${ }^{14} \mathrm{CO}_{2}$ desprendidos (28,5 e 5,0\%, respectivamente) que o observado neste trabalho. As elevadas taxas de degradação de atrazina descritas na literatura geralmente são obtidas em experimentos com pequena amostra de solo, em condições constantes de aeração e alto teor de umidade. Estas condições otimizam o processo de degradação e raramente representam a realidade ambiental (Winkelmann \& Klaine, 1991).
Os baixos níveis de mineralização da ${ }^{14} \mathrm{C}$-atrazina foram observados logo nos primeiros dias do experimento e mantiveram-se praticamente constantes, não apresentando picos de intensidade de mineralização (Figura 4). A ausência de uma fase lag na curva revela a existência de uma população microbiana possivelmente pequena, porém adaptada ao composto, produzindo enzimas de degradação. Em contrapartida, Queiroz (1997) e Langenbach et al. (2000) observaram uma fase crescente de liberação de ${ }^{14} \mathrm{CO}_{2}$ a partir do vigésimo dia de experimento, comportamento típico da degradação microbiana. Os baixos valores de degradação podem ser resultantes da presença de outras fontes de nutrientes, que tornam a atrazina dispensável aos microrganismos e, conseqüentemente, reduzem o processo de degradação biótica.

Experimentos de mineralização da atrazina por microrganismos têm demonstrado o aumento do metabolismo e liberação de ${ }^{14} \mathrm{CO}_{2}$ quando este herbicida é utilizado como única fonte de nitrogênio (Mandelbaum et al., 1995). Segundo alguns autores, a variação da intensidade de desprendimento de ${ }^{14} \mathrm{CO}_{2}$ em diferentes solos pode estar relacionada, principalmente, com as diferenças no teor e tipo de matéria orgânica (Nakagawa et al., 1995; Peixoto, 1998). De modo geral, a maior biomassa e a atividade microbiana relacionadas com degradação de herbidas estão associadas ao elevado teor de matéria orgânica do solo (Felsot \& Dzantor, 1990). Em contrapartida, a maior adsorção do herbicida à matéria orgânica reduz a biodisponibilidade e a biodegradação (Kontchou \& Gschwind, 1995; Langenbach et al., 2001).

Lixiviação de atrazina - Durante os 60 dias de experimento, num regime de chuva de $272 \mathrm{~mm}$ mês $^{-1}$, a lixiviação apresentou valores entre 4 e $11 \%$ nos quatro microcosmos (Figura 3). Esta variação entre os microcosmos deveu-se,

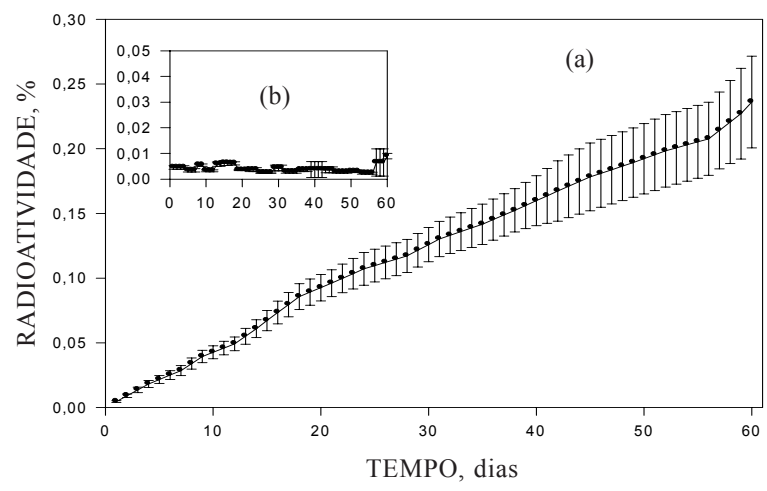

Figura 4. Percentagem de ${ }^{14} \mathrm{CO}_{2}$ liberado da degradação da atrazina nos 60 dias de experimento (média dos 4 microcosmos) $(a=\%$ acumulada, $b=\%$ diária). As barras verticais indicam o desvio-padrão. 
provavelmente, ao fluxo preferencial causado por fissuras no solo. A indicação do potencial de lixiviação pode ser avaliado no experimento de campo, onde a atrazina foi encontrada a $50 \mathrm{~cm}$ de profundidade após 90 dias de experimento, podendo ter alcançado maiores profundidades não estudadas neste trabalho (Figura 6).

A textura e o baixo teor de matéria orgânica deste solo (Quadro 1) diminuem a capacidade de retenção de água e favorecem a lixiviação da solução do solo com o arraste de material radioativo. Resultados similares foram obtidos por Lavy et al. (1973) em solos de textura arenosa, com baixo teor de matéria orgânica. Em comparação, experimentos sob condições similares, num Latossolo VermelhoAmarelo, tiveram lixiviação de aproximadamente $1 \%$ (Langenbach et al., 2000). A textura argilosa e o teor mais elevado de matéria orgânica permitem maior adsorção, reduzindo, assim, o movimento do herbicida. Se, por um lado, o fator de permeabilidade do solo se correlaciona com a lixiviação (Neurath et al., 2004); em contrapartida, o teor de matéria orgânica associada à adsorção destas moléculas pode sobrepor-se ao processo de lixiviação em períodos secos (Langenbach et al., 2001). A intensa lixiviação de atrazina neste solo pode significar o alcance de camadas mais profundas e o conseqüente risco de contaminação dos lençóis d'água.

Distribuição da atrazina no perfil do solo A maior parte da radioatividade aplicada ficou retida no solo (Figura 3), encontrando-se aproximadamente $75 \%$ do total aplicado nos primeiros $5 \mathrm{~cm}$ do solo e cerca de $15 \%$ a atrazina foi distribuída, de forma gradativa e decrescente, até os $25 \mathrm{~cm}$ de profundidade (Figura 5). Isto mostra que houve um movimento descendente da atrazina neste solo, visto que uma parte foi coletada no lixiviado. Esta distribuição

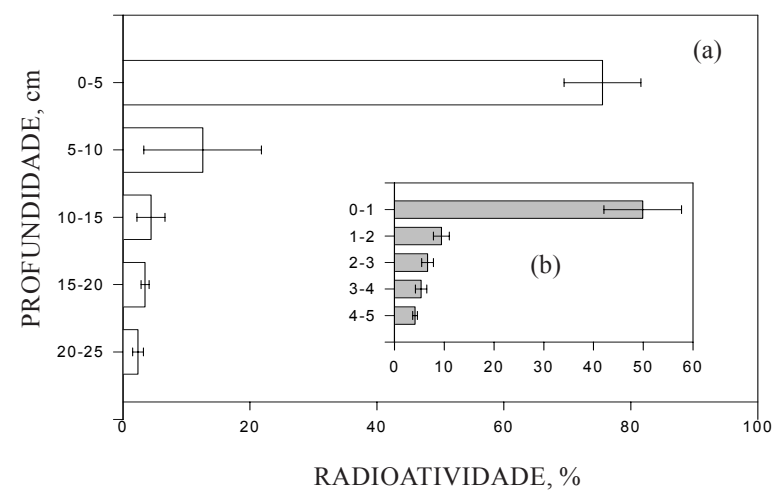

Figura 5. Distribuição de radioatividade em profundidade ( $\mathrm{a}=\%$ radioatividade até $0 \mathrm{~s} 25 \mathrm{~cm}$ de $5 \mathrm{em} 5 \mathrm{~cm}, \mathrm{~b}=\%$ radioatividade nos primeiros $5 \mathrm{~cm}$ ). As barras verticais indicam o desvio-padrão. deveu-se, provavelmente, ao teor de matéria orgânica presente nas camadas superficiais, a qual, mesmo sendo pequena, foi considerada como principal componente envolvido no processo de adsorção e retenção deste herbicida (Schiavon et al., 1990; Barriuso et al., 1991). A adsorção, no entanto, não descarta a possibilidade de retorno do composto para solução, propiciando a lixiviação, quando as forças envolvidas no processo são de natureza mais fraca.

A redução da biomassa microbiana e a formação de condições anaeróbias reduziram a degradação deste composto em camadas mais profundas, garantindo maior persistência no ambiente.

\section{Experimento de campo}

O herbicida atrazina aplicado na superfície foi encontrado ao longo do perfil do solo até $50 \mathrm{~cm}$, 90 dias após aplicação (Figura 6). Isto mostra que houve um movimento descendente da atrazina, tendo sido uma parte não medida lixiviada para camadas mais profundas. No período de 90 dias, percebeu-se diminuição gradativa de atrazina extraível, principalmente na camada superficial e no total encontrado no solo. Isto se explica pela possibilidade de lixiviação e por outros fatores, tais como: perdas por volatilização, mineralização, degradação e incorporação em resíduos ligados nãoextraíveis do solo. Estes parâmetros são de difícil determinação em experimentos de campo, razão pela qual foram determinados utilizando moléculas radiomarcadas em microcosmo (Schroll et al., 1994).

O deslocamento de atrazina por escorrimento superficial, apesar de pequeno, esteve entre os valores geralmente observados na literatura, de 1 a $5 \%$ (Schneider et al., 1997). A percentagem de atrazina encontrada dois dias após aplicação foi de

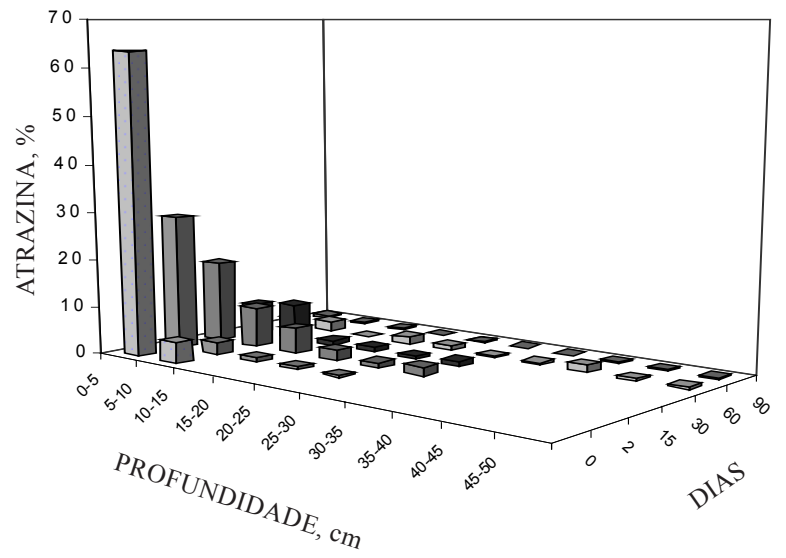

Figura 6. Distribuição da atrazina em diferentes profundidades do solo em 90 dias de experimento. 
$1,6 \%$ e, nos sedimentos erodidos, foi de $0,4 \%$, reduzindo-se, com o decorrer do tempo, a menos de um décimo destes valores, isto é $<0,2$ e $<0,05 \%$, respectivamente (Figuras 7 e 8). A quantidade de atrazina detectada no sedimento erodido foi aproximadamente 4 vezes inferior à encontrada na água de escorrimento. Esta quantidade corresponde às observações de Wauchope (1978), que encontrou maior quantidade de atrazina dissolvida na água (65 a $95 \%$ ) e muito pouco adsorvida no sedimento. As características físicas e químicas dos pesticidas podem indicar o potencial de perda destes produtos na fase solúvel ou adsorvidos em partículas de solo erodidos. Conforme sugerido por Racke (1990), pesticidas como a atrazina com solubilidade em água superior a $10 \mathrm{mg} \mathrm{L}^{-1}$ tendem a mover-se predominantemente na fase líquida, enquanto pesticidas menos solúveis tendem a mover-se principalmente adsorvidos em partículas de solo.

Neste trabalho, as maiores perdas por água de escorrimento ocorreram com as chuvas torrenciais logo após a aplicação do herbicida, um comportamento semelhante àqueles observados por outros autores (Belamie \& Gouy, 1992; Carluer et al., 1996; Patty et al., 1997). Por outro lado, a maior intensidade de chuvas pode promover perdas por lixiviação no solo, em detrimento do escoamento superficial. A redução das perdas também pode ser resultado da progressiva adsorção e penetração destes produtos no solo ao longo do tempo (Ma et al., 1993; Dur et al., 1998).

$\mathrm{O}$ alto teor de areia do Argissolo Vermelho-Amarelo (Quadro 1) pode representar menor potencial de adsorção de pesticidas e boa permeabilidade,

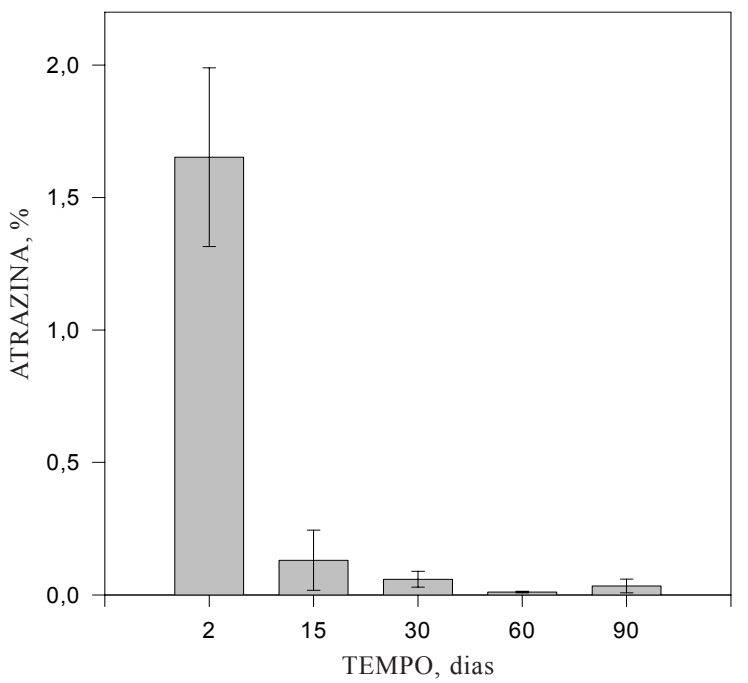

Figura 7. Percentagem de atrazina na água do escorrimento superficial. As barras verticais indicam o desvio-padrão (três repetições).

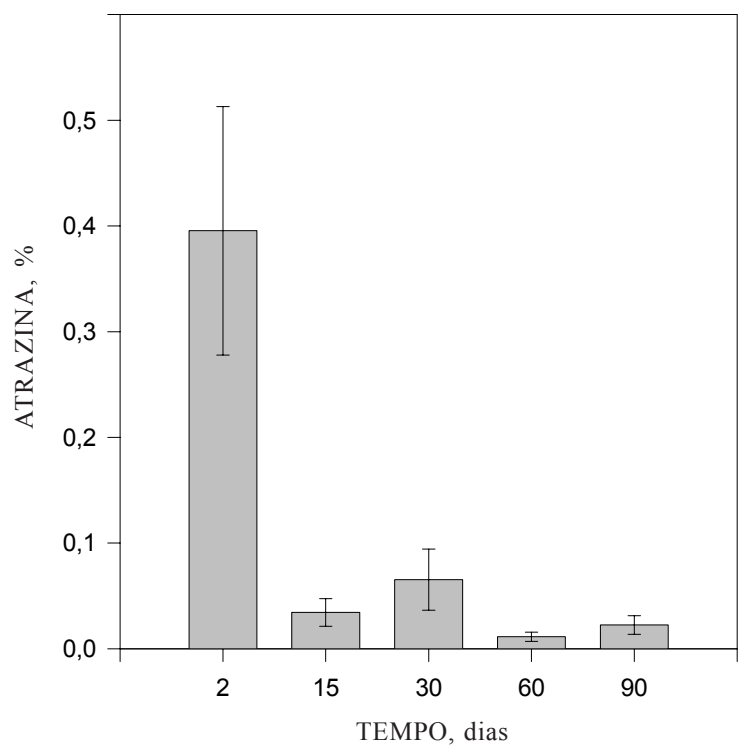

Figura 8. Percentagem de atrazina nos sedimentos carreados pelo escorrimento superficial. As barras verticais indicam o desvio-padrão.

facilitando o processo de lixiviação (Schneider et al., 1997). Experimentos similares em outros solos podem fornecer resultados muito distintos, demandando pesquisas específicas.

Os percentuais de carreamento de pesticidas por escorrimento superficial diminuem significativamente com o aumento da área agrícola, porém o aporte em valores absolutos aumenta (Schneider et al., 1997). Considerando o grande número de variáveis, avaliações são difíceis em razão de diferentes áreas, porém persiste o fato de que o escorrimento superficial, mesmo com valores relativamente baixos, é a maior fonte de poluição das águas superficiais por pesticidas.

Considerando a correlação entre a quantidade aplicada e a concentração de pesticidas nos rios das bacias hidrográficas (Lopez-Flores et al., 2003), o desafio para sustentabilidade ambiental é reduzir ao mínimo as doses utilizadas na agricultura. Outra abordagem é utilizar produtos com alta capacidade de adsorção e baixa solubilidade em água (Dur et al., 1998). A redução do escorrimento superficial também pode ser alcançada por valas cobertas com vegetação (Cooper et al., 2004). O trabalho de Hyer et al. (2001), utilizando a modelagem matemática na determinação de processos que controlam o escorrimento superficial, mostrou uma correlação positiva entre a quantidade de chuvas e a intensidade do escorrimento. Por fim, este trabalho mostrou que o fator tempo entre a aplicação e chuvas torrenciais é importante no processo de adsorção, motivo pelo qual se deve evitar a aplicação de agrotóxicos quando há previsão de chuvas fortes. 


\section{CONCLUSÕES}

1. O processo de mineralização mostrou-se reduzido na dissipação da atrazina neste solo, aumentando, conseqüentemente, sua persistência.

2. A perda de atrazina por lixiviação, tanto no microcosmo como em experimento de campo, revelou um risco de contaminação do lençol d'água em solos de textura média a arenosa.

3. A perda da atrazina por escorrimento superficial (1,6\%) foi quatro vezes maior do que a ocorrida pela adsorção nas partículas erodidas $(0,4 \%)$ em enxurradas, 2 dias após a aplicação do herbicida. Em intervalos de tempo acima de 15 dias, estes valores foram reduzidos a um décimo, mostrando a importância de evitar a aplicação do herbicida quando há previsão de chuvas.

\section{AGRADECIMENTOS}

Este trabalho contou com a valiosa colaboração do técnico Edir Martins Ferreira, para as análises de resíduos, e com a participação do Prof. Ravelli Neto da UFRRJ, para criar as condições locais ao experimento de campo. À Syngenta, por ceder gentilmente a molécula marcada com ${ }^{14} \mathrm{C}$ no anel da atrazina. Apoio financeiro foi obtido junto ao CNPq, FUJB e FAPERJ.

\section{LITERATURA CITADA}

ADAMS, C.D. \& THURMAN, E.M. Formation and transport of deethylatrazine in the soil and vadose zone. J. Environ. Qual., 20:1-54, 1991.

BALINOVA, A. \& BALINOV, I. Determination of herbicide residues in soil in the presence of persistent organochlorine insecticides. Fresenius J. Anal. Chem., 339:409-412, 1991.

BARRIUSO, E.; SCHIAVON, M.; ANDREUX, F. \& PORTAL, J.M. Localization of atrazine nonextractable (bound) residues in soil size fraction. Chemosphere, 22:1131-1140, 1991.

BELAMIE, R. \& GOUY, V. Introduction des pollutants dans le milieu fluvial. Influence du ruissellement des sols. Oceanis, 18:505-521, 1992.

BERTONI, J. \& LOMBARDI-NETO, F. Fatores que influem na erosão. In: BERTONI, J. \& LOMBARDI-NETO, F. Conservação do solo. 3.ed. São Paulo, Icone, 1993. p.45-67.

CARLUER, N.; GOUY, V.E. \& GRILL, J.N. Contamination des eaux de surface par des produits phyosanitaires et modélisation. Ingenieries-EAT, 6:19-30, 1996.

CLAY, S.A. \& KOSKINEN, W.C. Adsorption and desorption of atrazine, hydroxyatrazine, and s-glutathione atrazine on two soils. Weed Sci., 38:262-266, 1990.

CONNELL, W.D. Bioacumulation in xenobiotic compounds. In: CONNELL, W.D., ed. Evaluation of the bioconcentration factor, biomagnification factor, and related physicochemical properties of organic compounds. New York:CRC Press, 1990. p.9-45.
COOPER, C.M.; MOORE, M.T.; BENNETT, E.R.; SMITH, S.; FARRIS, J.L.; MILAM, C.D. \& SHIELDS, F.D. Innovative uses of vegetated drainage ditches for reducing agricultural runoff. Water Sci. Tecnol., 49:117-123, 2004.

DAY, P.R. Particle fractionation and particle-size analysis. In: Methods of soil analysis. Madison: American Society of Agronomy, 1965. p.545-566.

DÖRFLER, U.; FEICHT, E.A. \& SCHEUNERT, I. S-Triazine residues in groundwater. Chemosphere, 35:99-106, 1997.

DÖRFLER, U.; HAALA, R.; MATHIES, M. \& SCHEUNERT, I. Mineralization kinetics of chemicals in soils in relation to environmental conditions. Ecotox. Environm. Safety 34:216-222, 1996.

DUR, J.-C.; GOUY, V.; CALVET, R.; BELAMIE, R. \& CHAPLAIN, V. Influence of adsorption-desorption phenomena on pesticide runoff measured under controlled conditions. C. R. Acad. Sci., 327:405-411, 1998.

EMPRESA BRASILEIRA DE PESQUISA AGROPECUÁRIA EMBRAPA. Centro Nacional de Pesquisa em Solos. Manual de métodos de análise de solo. 2.ed. Rio de Janeiro, 1997. $212 \mathrm{p}$.

FELSOT, A.S. \& DZANTOR, E.K. Enhancing biodegradation for detoxification of herbicide waste in soil. In: RACKE, K.D. \& COATS J.R., eds. Enhanced biodegradation of pesticides in the environment. Washington, DC: ACS, 1990. p.249-268. (ACS Symposium Series, 426)

GAYNOR, J.D.; MACTAVISH, D.C. \& FINDLAY, W.I. Surface and sub-surface transport of atrazine and alachlor from a Brookston clay loam under continuous corn production. Arch. Environ. Contam. Technol., 23:240-245, 1992.

GLOTFELTY, D.E.; LEECH, M.M.; JERSEY, J. \& TAYLOR, A.W. Volatilization and wind erosion of soil surface applied atrazine, simazine, alachlor and toxaphene. J. Agric. Food Chem., 37:546-551, 1989 .

GLOTFELTY, D.E.; TAYLOR, A.C.; TURNER, C. \& ZOLLER, W.H. Volatilization of surface-applied pesticides from fallow soil. J. Agric. Food Chem., 32:638-643, 1984.

GOEDERT, W.J. Management of the Cerrado soils of Brazil: a review. J. Soil Sci., 34:405-428, 1983.

HAN, S.O. \& NEW, P.B. Effect of water availability on degradation of 2,4-dichlorphenoxyacetic acid (2,4-D) by soil microorganisms. Soil Biol. Biochem., 26:1689-1697, 1994.

HERNANI, L.C.; SALTON, J.C.; FABRÍCIO, A.C; DEDECEK, R. \& ALVES-JÚNIOR, E. M. Perdas por erosão e rendimento de soja e de trigo em diferentes sistemas de preparo de um Latossolo Roxo de Dourados (MS). R. Bras. Ci. Solo, 21:667-676, 1997.

HUANG, L. \& PIGNATELLO, J. Improved extraction of atrazine and metolachlor in field soil samples. J. Assoc. Off. Anal. Chem., 73:443-446, 1990.

HYER, K.E.; HORNBERGER, G.M. \& HERMAN, J.S. Processes controlling the episodic streamwater transport of atrazine and other agrichemicals in na agricultural watershed. J. Hidrol., 254:47-66, 2001.

INSTITUTO BRASILEIRO DE MEIO AMBIENTE - IBAMA. Manual de testes para avaliação da ecotoxicidade de agentes químicos. 2.ed. Brasília, 1990. 351p. 
KONTCHOU, C.Y. \& GSCHWIND, N. Mineralization of the herbicide atrazine in soil inoculated with a Pseudumonas strain. J. Agric. Food Chem., 43:2291-2294, 1995.

KREUGER, J. Pesticides in stream water within an a agricultural catchment in southern Sweden, 1990-1996. Sci. Total Environ., 216:227-278, 1998.

LAABS, V.; AMELUNG, W.; PINTO, A.; ALTSTAEDT, A.\& ZECH, W. Leaching and degradation of corn and soybean pesticides in an Oxisol of the Brazilian Cerrados. Chemosphere, 41:1441-1449, 2000.

LANGENBACH, T.; SCHROLL, R. \& PAIM, S. Fate and distribution of ${ }^{14} \mathrm{C}$-atrazine in a tropical oxisol. Chemosphere, 40:449-455, 2000.

LANGENBACH, T.; SCHROLL, R. \& SCHEUNERT, I. Fate of the ${ }^{14} \mathrm{C}$-terbuthylazine in Brazilian soils under various climatic conditions. Chemosphere, 45:387-398, 2001.

LAVY, T.L.; ROETH, F.W. \& FENSTER, C.R. Degradation of 2,4-D and atrazine at three soil depths in the field. J. Environ. Qual., 2:132-137, 1973.

LERCH, R.N. \& BLANIHARD, P.E. Watershed vulnerability to herbicide transport in northern Missouri and southern Iowa streams. Environ. Sci. Technol., 37:5518-5527, 2003.

LOPEZ-FLORES, R.; QUINTANA, X.D.; SALVADÓ, V.; HIDALGO, M.; SALA, L. \& MORENO-AMICH, R. Comparation of nutrient and contaminant fluxes in two areas with different hydrological regimes (Emporda Wetlands, NE Spain). Water Res., 37:3034-3046, 2003.

MA, L.; SOUTHWICK, L.M.; WILLIS, G.H. \& SELIM, H.M. Hysteretic characteristics of atrazine adsorption-desorption by a Sharkey soil. Weed Sci., 41:627-633, 1993.

MANDELBAUM, R.T.; ALLAN, D.L. \& WACKETT, L.P. Isolation and characterization of the Pseudomonas spp. that mineralizes the s-triazine herbicide atrazine. Appl. Environ. Microbiol., 61:1451-1457, 1995.

NAKAGAWA, L.E.; LUCHINI, L.C.; MUSUMECI, M.R. \& ANDRÉA, M.M. Comportamento da atrazina em solos brasileiros em condições de laboratório. Pesq. Agropec. Bras., 30:471-476, 1995.

NEURATH, S.K.; SADEGHI, A.M.; SHIRMOHAMMADI, A.; ISENSEE, A.R. \& TORRENTS, A. Atrazine distribution measured in soil and leachate following infiltration conditions. Chemosphere, 54:489-496, 2004.

ÖSTERREICHER-CUNHA, P.; LANGENBACH, T.; TORRES, J.P.M.; LIMA, A.L.C.; CAMPOS, T.M.P.; VARGAS, E.A. \& WAGENER, A.R. HCH distribution and microbial parameters after liming of a heavily contaminated soil in Rio de Janeiro. Environ. Res., 93:316-327, 2003.

PATTY, L.; REAL, B.E. \& GRILL, J.J. The use of grassed buffer strips to remove pesticides, nitrates and soluble phosphorus compounds from runoff water. Pestic. Sci., 49:243-251, 1997.

PEIXOTO, M.F.S.P. Distribuição do herbicida ${ }^{14} \mathrm{C}$-atrazina na matéria orgânica de dois solos e remobilização de seus resíduos ligados em ácidos fúlvicos. Piracicaba, Universidade de São Paulo, 1998. 80p. (Tese de Doutorado)

POLKOWSKA, Z.; GÓRECKI, T. \& NAMIESNIK, J. Quality of roof runoff waters from an urban region. Chemosphere, 49:1275-1283, 2002.

QUEIROZ, B.P.V. Biodegradação de ${ }^{14} \mathrm{C}$-atrazina em condições semi controladas. Piracicaba, Universidade de São Paulo, 1997. 95p. (Tese de Mestrado)
RACKE, K.D. Pesticide in the soil microbial ecosystem. In: RACKE, K.D. \& COATS, J.R., eds. Enhanced biodegradation of pesticides in the environment. Washington, ACS, 1990. p.1-12. (ACS Symposium Series, 426)

RAMOS, D.P.; CASTRO. A.F. \& CAMARGO, M.N. Levantamento detalhado de solos da área da Universidade Federal Rural do Rio de Janeiro. Pesq. Agropec. Bras., Ser. Agron., 8:1-27, 1973.

REICHENBERG, S.; AMELUNG, W.; LAABS, V.; PINTO, A.; TOTSSCHE, K.U. \& ZECH, W. Pesticide displacement along preferencial flow pathways in a Brazilian Oxisol. Geoderma, 110:63-86, 2002.

ROY, W.R. \& KRAPAC, I.G. Adsorption and desorption of atrazina and deethylatrazine by low organic carbon geologic materials. J. Environ. Qual., 23:549-556, 1994.

SCHIAVON, M.; BARRIUSO, E.; PORTAL, J.M.; ANDREUX, F.; BASTIDE, J.; COSTE, C. \& MILLET, A. Etude du devenir de deux substances organiques utilisées dans les sols, I' une massivement (I' atrazine) et I' autre à I' etat de trace (le metsulfuron-méthyl), à I' aide de molécules marquées au ${ }^{14} \mathrm{C}$. SRETIE/MERE, 7219. opération 23701 87 40131. France, 1990.

SCHNEIDER, M.; HERTL, P. \& DÜFER, B. Runoff of plant protection products from agricultural areas - An evaluation of literature and reflection. In: Pflanzenschutzmitteleinträge in Oberflächengewässer durch Runoff und Dränung. Ed. Berlin, Biologische Bundesanstalt für Land und Forstwirtschaft. 1997. p.6386.

SCHROLL, R.; CAO, G.; LANGENBACH, T. \& SCHEUNERT, I. "Microcosm"- a test system to determine the fate of pesticides in undisturbed soil columns. In: COPIN, A.; HOUINS, G.; PUSSEIMIER, L. \& SALAMBIER, J.F., eds. Environmental behaviour of pesticides and regulatory aspects. Rixensart, European Study Service, Rixensart, 1994. p.236-241.

SCHROLL, R.; LANGENBACH, T.; CAO, G.; DÖRFLER, U.; SCHNEIDER, P. \& SCHEUNERT, I. Fate of $\left({ }^{14} \mathrm{C}\right)$ terbutylazine in soil-plant systems. Sci. Total Environ., 123/124:377-389, 1992.

SEILER, A.; BRENNEISEN, P. \& GREEN, D.H. Benefits and risks of plant protection products possibilities of protecting drinking water:case atrazine. Water Supply, 10:31-42, 1992.

SINDAG. Estatisticas de consumo de defensivos agrícolas no Brasil. São Paulo, 1998. não paginado.

SOLOMON, K.R.; BAKER, D.B.; RICHARDS, R.P.; DIXON, K.R.; KLAINE, S.J.; LA POINT, T.W.; KENDAL, R.J.; WEISSKOPF, C.P.; GIDDINGS, J.M.; GIESY, J.P.; HALL, L.W. \& WILLIAMS, W.M. Ecological risk assessment of atrazine in North American surface waters. Environ. Toxicol. Chem., 15:31-76, 1995.

USDA. Soil Conservation Service, Soil Survey Stalf. Soil Taxonomy-A basic system of soil classification for making and interpreting soil surveys. Agriculture Handbook, Washington, DC, 1975. 436p.

WAUCHOPE, R.D. The pesticide content of surface water draining from agricultural fields - a review. J. Environ. Qual., 7:459-472, 1978.

WINKELMANN, D.A. \& KLAINE, S.J. Degradation and bound residue formation of atrazine in Western Tennessee soil. Environ. Toxicol. Chem., 10:335-345, 1991. 